Recherches en didactique des langues et des cultures

Les cahiers de l'Acedle

$13-2 \mid 2016$

Interactions langagières et didactique des langues

\title{
Faire parler l'apprenant en classe de FLE
}

Le rôle et l'influence des indices prosodiques

Malak Moustapha Sabeur

\section{OpenEdition}

Journals

Édition électronique

URL : http://journals.openedition.org/rdlc/826

DOI : $10.4000 /$ rdlc. 826

ISSN : $1958-5772$

Éditeur

ACEDLE

Référence électronique

Malak Moustapha Sabeur, "Faire parler l'apprenant en classe de FLE », Recherches en didactique des langues et des cultures [En ligne], 13-2 | 2016, mis en ligne le 25 septembre 2016, consulté le 30 avril 2019. URL : http://journals.openedition.org/rdlc/826; DOI : 10.4000/rdlc.826

Ce document a été généré automatiquement le 30 avril 2019.

\section{(c) $(1)$}

Recherches en didactique des langues et des cultures is licensed under a Creative Commons AttributionNonCommercial-NoDerivatives 4.0 International License 


\section{Faire parler l'apprenant en classe de FLE}

Le rôle et l'influence des indices prosodiques

Malak Moustapha Sabeur

\section{Introduction}

1 Le présent article propose de souligner le rôle et l'influence des indices prosodiques et gestuels présents dans la parole de l'enseignant sur les productions verbales et les réponses des apprenants en classe de français langue étrangère. Les recherches en didactique des langues portant sur les interactions (Cicurel, 1985 ; 2011) attribuent à l'enseignant un rôle considérable dans le contrôle de la parole de l'apprenant. Selon Cicurel :

Le partenaire enseignant exerce sur le partenaire enseigné un certain contrôle, celui de l'exercice de la parole, c'est lui qui l'attribue et c'est lui qui fait un commentaire sur la parole produite(Cicurel, $2011: 29$ ).

2 Notre recherche s'intéresse spécifiquement au rôle de la manière de parler de l'enseignant. Si l'apprentissage se révèle partiellement dans la parole des apprenants (quand il s'agit de répondre à une question) et s'effectue en partie dans les interactions (Cicurel, 2011), c'est parce que l'enseignant utilise des outils divers parmi lesquels nous retenons pour le présent article les outils prosodiques et gestuels. Dans la parole, même si les facteurs qui contribuent à la compréhension (prosodie, geste etc.) sont souvent associés (Colleta, 2005), il arrive que nous les séparions dans le présent article pour une analyse fine de l'influence de chacun.

\section{Objectifs de la recherche}

Caelen Haumont (2006) a étudié dans la parole spontanée les fonctions pragmatiques de la prosodie: elle distingue d'un côté le "faire savoir» (le «faire entendre» et le «faire comprendre» auxquelles correspondent respectivement les fonctions prosodiques 
(démarcative et discriminative) et, d'un autre côté, le "faire croire ». "Faire savoir " étant un but majeur de l'enseignement, nous insistons dans le présent article sur «la fonction discriminative» de la prosodie dans la parole de l'enseignant qui agit sur le contenu des paroles des apprenants. Mais, à part les demandes directes (parlez, répondez à la question suivante, encore, etc.), la manière de parler et les gestes incitent les apprenants à prendre la parole en leur offrant des indices les orientant vers la réponse souhaitée.

4 Cependant, la manière de parler n'est pas toujours spontanée (le cas de la focalisation prosodique voulue par exemple). Si l'enseignant se rend compte de son impact qui peut parfois être négatif sur l'apprentissage, il contribue, tout en la contrôlant, à favoriser indirectement l'apprentissage et l'autonomie de l'apprenant.

5 Le premier objectif du présent article consiste ainsi à montrer un exemple de focalisation prosodique qui peut avoir un "effet pervers » sur l'apprentissage. D'un autre côté, les recherches menées sur le rôle du geste en classe de FLE (Tellier, 2008: 40) considèrent ce dernier comme un " geste pédagogique » qui informe, évalue et anime.

6 Le second objectif de notre article consiste à montrer, spécifiquement et par une analyse fine, le rôle du geste de l'enseignant qui fait parler et qui oriente les réponses des apprenants lors d'une interaction et ce, avec un usage associé de la voix leur permettant de comprendre les demandes implicites.

\section{Les demandes implicites de l'enseignant}

7 En analysant les interactions en classe de langue, Cicurel (2011:38) distingue des actes de paroles qui sous-tendent des demandes de parler diverses (parler, parler de, parler encore, parler mieux etc.). Mais les demandes de l'enseignant ne sont pas toujours connues ou seulement devinées à partir de la structure superficielle des phrases comme par exemple le cas de la question « il y a qui ? » où la question sous-tend une demande de «parler de » ou bien « un autre mot que » où le mot «autre » signifie «parler mieux». Les marqueurs de structuration de la conversation (les ligateurs et les ponctuants) distingués par Morel et Danon Boileau (1998) (oui, hein, bon, etc.), peuvent signifier à chaque fois une demande différente selon la manière avec laquelle ils ont été produits avec les gestes qui leur sont associés (gestes des mains, regard, mimique faciale etc.). Les interactions didactiques sont des interactions sociales mais spécifiques "par le cadre participatif (un professeur et des élèves) » (Cicurel, 2011: 19), où geste et parole sont associés. Le geste de l'enseignant est généralement un geste pédagogique, d'après Tellier (2008: 41), qui « consiste en un geste des bras et des mains (mais il peut aussi être composé de mimiques faciales) utilisé par l'enseignant de langue dans un but pédagogique». Nous verrons dans le présent article les intentions de l'enseignant et les mêmes demandes de faire parler, parler mieux, parler encore, ne parler plus, mais aussi de parler haut (Moustapha Sabeur, 2008), compris par les apprenants grâce aux indices prosodiques et gestuels.

8 Selon Gumperz (1989: 28), pour comprendre l'intention du locuteur et avancer dans l'échange verbal, il faut des indices de «contextualisation» ou de «signalement » connus par les deux partenaires de la communication, indices en rapport avec ses «attentes stéréotypées de cooccurrence entre le contenu et le style superficiel».

9 Les indices de contextualisation sont des «caractéristiques superficielles» propres à la forme du message. Ils constituent l'outil par lequel les locuteurs signalent et les 
allocataires interprètent la nature de l'activité en cours, la manière dont chaque phrase se rapporte à ce qui précède ou à ce qui suit. Gumperz (1989) distingue, dans la parole, plusieurs éléments permettant de connaître les intentions de communication du locuteur parmi lesquels il note les éléments prosodiques. Le contexte didactique manifeste des tâches interactionnelles (comprises par les apprenants en tant qu'intention de communication)au moyen d'indices particuliers, parmi lesquels la manière de parler et les gestes de l'enseignant comme le précise Cicurel :

Ce sont généralement l'intonation et la mimique accompagnant l'énoncé qui permettent aux apprenants d'interpréter l'intention de l'enseignant(Cicurel, 1985 :

78).

L'influence de la structuration mélodique d'un énoncé sur la perception d'un auditeur est expliquée par Caelen Haumont en posant la relation de faire-savoir (du côté du locuteur) et comprendre (du côté de l'auditeur):

Cette structuration est distinctive dans la mesure où elle fournit au niveau le plus élevé, des indications qui permettent côté locuteur de faire-savoir, et côté auditeur de comprendre, si par exemple le locuteur asserte un énoncé, pose une question, donne un ordre, s'étonne ou s'exclame. (2006: 192)

11 Dans quelle mesure les intentions de communication de l'enseignant sont connues et reconnues par les apprenants à partir de sa manière de parler et de ses gestes? Par exemple, sauront-ils si une accélération du rythme de la parole signifie que leur enseignant se dépêche ou qu'il veut passer brièvement sur une idée ? Ou encore, saurontils dans le cas inverse, si un ralentissement du rythme de sa parole signifie qu'il veut focaliser une expression importante ou bien qu'il prend le temps nécessaire pour formuler ses idées? Il revient à l'enseignant d'adopter certaines stratégies.

Selon Cicurel (2002 : 148), la place qu'occupe l'enseignant « exige qu'il fasse appel à diverses stratégies pour se faire comprendre, pour favoriser l'apprentissage, pour attirer l'attention sur les problèmes langagiers ». Mais les apprenants répondent-ils parfois correctement à une question parce qu'ils ont compris ou bien parce qu'ils étaient dirigés et guidés par des indices (vocaux et/ou gestuels) ? C'est une question qui échappe souvent aux enseignants et que nous soulignons dans le présent article au moyen de quelques extraits de séquences enregistrées, transcrites et filmées.

\section{Méthodologie et corpus}

13 L'étude consiste à trouver des éléments d'intonation et/ou de geste qui apparaissent dans deux corpus différents afin d'observer et d'illustrer le rôle et l'influence des indices prosodiques et gestuels sur les productions verbales des apprenants. Les corpus sont constitués à partir de deux activités différentes (compréhension écrite et compréhension orale). Le tableau 1 présente le contexte dans lequel ont eu lieu les observations et les enregistrements. Les classes choisies dépendent de l'enseignant qui propose d'être observé dans telle ou telle classe.

\section{Description du contexte et des circonstances des enregistrements}

14 Il s'agit de deux corpus recueillis à partir d'enregistrements effectués pendant deux périodes différentes. Nous notons que les méthodes d'observation ne sont pas les mêmes pour les deux corpus. 
Tableau 1 - Les circonstances des enregistrements des deux corpus

\begin{tabular}{|c|c|c|c|}
\hline & & Corpus 1 & Corpus 2 \\
\hline \multirow{7}{*}{$\begin{array}{l}\text { CONTEXTE } \\
\text { INSTITUTIONNEL }\end{array}$} & Institution & Lycée public & $\begin{array}{l}\text { Établissement } \\
\text { universitaire public }\end{array}$ \\
\hline & $\begin{array}{l}\text { Intitulé } \\
\text { du cours }\end{array}$ & Langue française & $\begin{array}{l}\text { Langue et civilisation } \\
\text { française }\end{array}$ \\
\hline & Activité & Compréhension écrite & Compréhension orale \\
\hline & Support du cours & Le manuel scolaire officiel & $\begin{array}{l}\text { chanson "c'est pas moi } \\
\text { c'est les autres " de Abdel } \\
\text { Malik. }\end{array}$ \\
\hline & $\begin{array}{l}\text { Date et Horaire de } \\
\text { l'observation }\end{array}$ & $\begin{array}{l}14 \text { avril } 2003 \text { de } 8 \mathrm{~h} \text { à } 8 \mathrm{~h} 55 \mathrm{du} \\
\text { matin }\end{array}$ & $\begin{array}{l}8 \text { avril } 2012 \text { à } 8 \text { h30 du } \\
\text { matin. }\end{array}$ \\
\hline & $\begin{array}{l}\text { Date et Horaire de } \\
\text { l'entretien d'auto- } \\
\text { confrontation }\end{array}$ & --- & 12 avril 2012. \\
\hline & $\begin{array}{lrr}\text { L'arrêt } & d u & \text { film } \\
\text { visionné } & \text { pour } \\
\text { s'auto-commenter }\end{array}$ & --- & $\begin{array}{l}\text { est effectué par } \\
\text { l'enseignante tout au } \\
\text { long de l'entretien }\end{array}$ \\
\hline $\begin{array}{l}\text { CONTEXTE } \\
\text { INTERACTIONNEL }\end{array}$ & Le public & $\begin{array}{l}\text { Classe de EB8 }\left(4^{\text {ème}}\right) \\
\text { composée de } 21 \text { apprenants } \\
(12 \text { filles et } 9 \text { garçons }) \text { et } \\
\text { ayant } 14 \text { à } 16 \text { ans. } \\
\text { L'enseignant est libanais, } 30 \\
\text { ans d'expérience. }\end{array}$ & $\begin{array}{l}\text { Étudiants en } 1^{\text {ère }} \text { année } \\
\text { licence (Langue et } \\
\text { civilisation française). } \\
\text { L'enseignanter est } \\
\text { tunisienne, } 30 \quad \text { ans } \\
\text { d'expérience. }\end{array}$ \\
\hline
\end{tabular}

Le premier, constitué en 2003, n'a pas été filmé mais retenu pour le présent article afin de souligner l'effet pervers de la focalisation prosodique lors d'une activité de compréhension écrite. Les gestes associés ne sont pas pris en compte étant donné que le cours n'a pas été filmé.

La méthodologie de recueil des données étant plus riche pour le second corpus constitué en 2012, elle consiste à filmer le cours et à le suivre d'un entretien d'auto-confrontation ${ }^{1}$. L'enseignante, de nationalité tunisienne, a été enregistrée et filmée en cours trois jours auparavant. Elle assure un cours de français langue étrangère pour la première année universitaire. Le choix de l'enseignante ne tient pas à la nature de sa voix, mais à ses compétences professionnelles et à sa capacité à réfléchir explicitement à ses pratiques d'enseignement. Il s'agit, en fait, d'une collègue (professeur universitaire) qui a 30 ans d'expérience dans le domaine de l'enseignement (au collège, au lycée puis à l'université) et qui a accepté d'être enregistrée et filmée en cours. Elle a accepté des auto-commenter tout en visionnant le film après coup. 
17 Afin de transcrire les séquences enregistrées, nous avons adopté un code consistant en des options conventionnelles développées par le Diltec (Laboratoire Didactique des langues des textes et des cultures) et d'autres spécifiques pour l'analyse de la prosodie (Tableau 2).

\section{Le code transcriptif adopté}

Dans le corpus1, l'enseignante tend à lire et à faire comprendre une phrase d'un texte du manuel scolaire : « elle apportait de l'or et elle venait demander à mon père de le transformer en bijou », (lecture + question de compréhension). Cette lecture (effectuée par l'enseignante) de type explicative sera comparée à la lecture magistrale de la même phrase (où l'enseignant n'a pas l'intention de poser des questions de compréhension mais de montrer seulement un modèle de lecture).

Tableau 2 - Le code transcriptif adopté

\begin{tabular}{|c|c|c|}
\hline \multirow{3}{*}{$\begin{array}{l}\text { Options conventionnelles } \\
\text { développées } \\
\text { Par le Diltec }\end{array}$} & Oui... & demande d'achèvement \\
\hline & P & enseignant \\
\hline & A & apprenant \\
\hline \multirow{7}{*}{ Code spécifique } & BI & accent d'intensité \\
\hline & BI JOU & accent de durée \\
\hline & $\begin{array}{l}\text { elle } \\
\text { apportait }\end{array}$ & lecture à haute voix \\
\hline & + & pause silence de courte durée \\
\hline & $\begin{array}{l}\text { transformer } \\
\text { transformer }\end{array}$ & $\begin{array}{l}\text { débit normal (taille de caractère } 12 \text { ) } \\
\text { débit ralenti avec allongement des syllabes (taille de } \\
\text { caractère 14) }\end{array}$ \\
\hline & $\uparrow$ & $\begin{array}{l}\text { continuation de l'intonation sur la dernière syllabe } \\
\text { devant une pause ou en fin d'un tour de parole }\end{array}$ \\
\hline & $\downarrow$ & $\begin{array}{l}\text { conclusion de l'intonation sur la dernière syllabe } \\
\text { devant une pause ou en fin d'un tour de parole }\end{array}$ \\
\hline
\end{tabular}

\section{Le contenu du corpus 1}

Séquence (1) : transcription d'une phrase lue lors de la lecture magistrale

$\mathrm{P}$ elle apportait de l'or $\uparrow$ + et elle venait demander à mon père de le transformer en Bijou $\downarrow$

Séquence (2) : transcription de la même phrase lue lors de la lecture explicative avec l'interaction qui suit : 
$01 \mathbf{P}+++$ Elle apportait de l'or et elle venait demander à mon père de le transformer en +BI-JOU $\downarrow++$ donc $\uparrow+$ que vient faire la femme ?que venait faire la femme $\uparrow+$ chez $\uparrow+$ le père du narrateur + + ?

02 A elle transformait en bijou $\downarrow$

03 As Elle transformait en bijou $\downarrow$

$04 \mathbf{P}$ Elle venait pourquoi faire ?

05 A transformer en bijou $\downarrow$

$06 \mathbf{P}$ AH pour transformer l'or... en quoi?

07 A en bijou $\downarrow$ (bruit)

08 P BON + BON $\downarrow++$ euh euh Mahmoud $\downarrow+$ que veut dire bijou ? qu'est-ce que c'est qu'un bijou?+

\section{Le contenu du corpus 2}

19 Dans le corpus2, il s'agit de montrer que pour enchaîner les interactions verbales, les demandes de parler sont facilitées par des usages différents de la voix et du geste et ce en produisant le même mot « pardon ».

\section{Séquence (1) transcrite}

$01 \mathrm{P}$ tu as retenu qu'il a changé de nom + qu'est-ce que vous avez retenu les autres?

02 A c'était un voleur

$03 \mathrm{P}$ pardon?

04 A c'était un voleur

\section{Séquence (2) transcrite:}

01 P elle a proposé de l'encadrer dans une école

$02 \mathrm{P}$ j'ai pas entendu

$03 \mathrm{~A} \mathrm{xx}$

04 P j'ai j'ai pas entendu

05 A à la rue

06 P A LA RUE + OUI alors

07 A populaire

$08 \mathrm{P}$ pardon?

09 A populaire

$10 \mathrm{P}$ populaire $\downarrow$ oui

11 A familial

$12 \mathrm{P}$ pardon?

13 A familier une chanson familière

$14 \mathrm{P}$ ah une chanson familière est-ce que ça veut dire

15 A engagé

$16 \mathrm{P}$ pardon?

17 A engagé

18 P EN-GA-GE + + très bien + donc c'est une musique + engagée et s'engage sur quel + + à quel niveau?

19 A social

20 P social $\downarrow$ donc c'est une musique + + engagé

Séquence (3) transcrite :

$01 \mathrm{P}$ quelle est la préoccupation des slameurs quelle est la préoccupation + + des rappeurs + alors est-ce qu'ils résolvent des problèmes?

02 A ils xx

03 P ils $\uparrow$... 
$04 \mathrm{~A}$ ils exploitent le problème

$05 \mathrm{P}$ i i ils ils $\uparrow$...

$06 \mathrm{~A}$ ils dévoilent

$07 \mathrm{P}$ ils dévoilent ils $\uparrow$...

$08 \mathrm{~A} \mathrm{xx}$

09 A ils militent

\section{La focalisation prosodique lors de la lecture explicative : analyse du corpus (1)}

20 Nous désignons par « lecture explicative » la lecture d'un texte dans le but d'expliquer et de poser des questions de compréhension. Par contre la lecture magistrale consiste en une lecture effectuée par l'enseignant dans le seul but de montrer un exemple de lecture.

\section{Une stratégie d'aide à prendre la parole}

21 La focalisation est une stratégie de mise en relief qui consiste à jouer volontairement sur les variations de débit, de durée, d'intensité, de fréquence et sur les silences. Elle permet, en mettant en relief la partie dominante sélectionnée du message à transmettre, d'éviter une mauvaise interprétation du message. Il s'agit d'une stratégie qui consiste en un passage d'une " action vocale inconsciente ou involontaire à une mimique vocale, consciente dans le cadre d'une interaction" (Guimbretière 2000: 299). En classe, la volonté de chaque enseignant intervient dans le choix du moment, de la partie à focaliser, du degré d'accentuation, de l'intensité qu'il prend en parlant, de son rythme de parole etc. Donc, si chaque enseignant possède des manières de parler socialement connues par ses apprenants, il a ses propres manières qu'il crée ou qu'il produit en situation, selon sa volonté ou non d'agir par sa voix.

Utiliser une voix consiste en des variations prosodiques stratégiques (Guimbretière, 2000), donc voulues, du débit (durée des syllabes) de l'énergie (intensité), de la mélodie (la fréquence) pour "afficher une attitude ou induire une action auprès de ses interlocuteurs " (Mousapha Sabeur \& Aguilar, 2014 : 70).

\section{La focalisation prosodique à fonction discriminative comme indice de réponse}

L'enseignant (corpus 1) effectue une lecture linéaire d'un texte choisi du manuel scolaire où après chaque phrase lue il pose une question de compréhension. Il adopte une stratégie de mise en relief, de focalisation d'un groupe de mots qui devrait constituer la réponse des apprenants à la question posée juste après la lecture. Pour Caelen Haumont :

[...] la prosodie, et au premier chef la mélodie, exercerait [...] dans l'expression cognitive du sens, non seulement une fonction démarcative mais aussi une fonction discriminative qu'en sélectionnant certaines unités parmi d'autres traduirait l'interprétation profonde du message (Caelen Haumont, 2006 : 193).

La manière de parler de l'enseignant (lors de la lecture d'une phrase d'un texte/corpus 1) aide les apprenants à discriminer la réponse avant qu'il ne la pose. La phrase: «Elle apportait de l'or et elle venait demander à mon père de le transformer en bijou » a été lue à deux moments : la lecture magistrale et la lecture explicative. 
Afin d'illustrer le phénomène de la focalisation nous comparons deux figures prises du logiciel Praat montrant la lecture magistrale (Figure 1) et la lecture explicative (Figure 2), de la même phrase. Nous remarquons qu'une continuité intonative caractérise la première alors qu'une variation prosodique subite a été effectuée dans la deuxième, sur la partie «le transformer en bijou » (diminution de l'intensité et allongement de la durée des syllabes). Il suffit d'observer la forme du signal sonore ${ }^{2}$ dans les deux figures (partie supérieure de la figure) pour saisir la différence.

Figure 1 - La phrase lue lors de la lecture magistrale

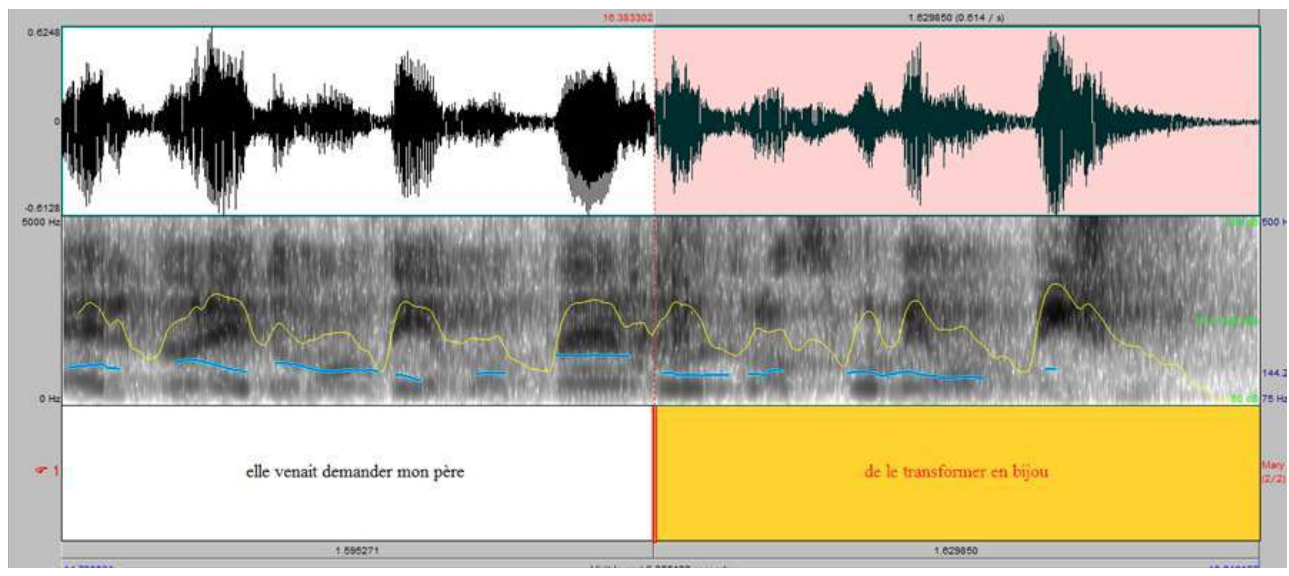

Figure 2 - La phrase lue lors de la lecture explicative

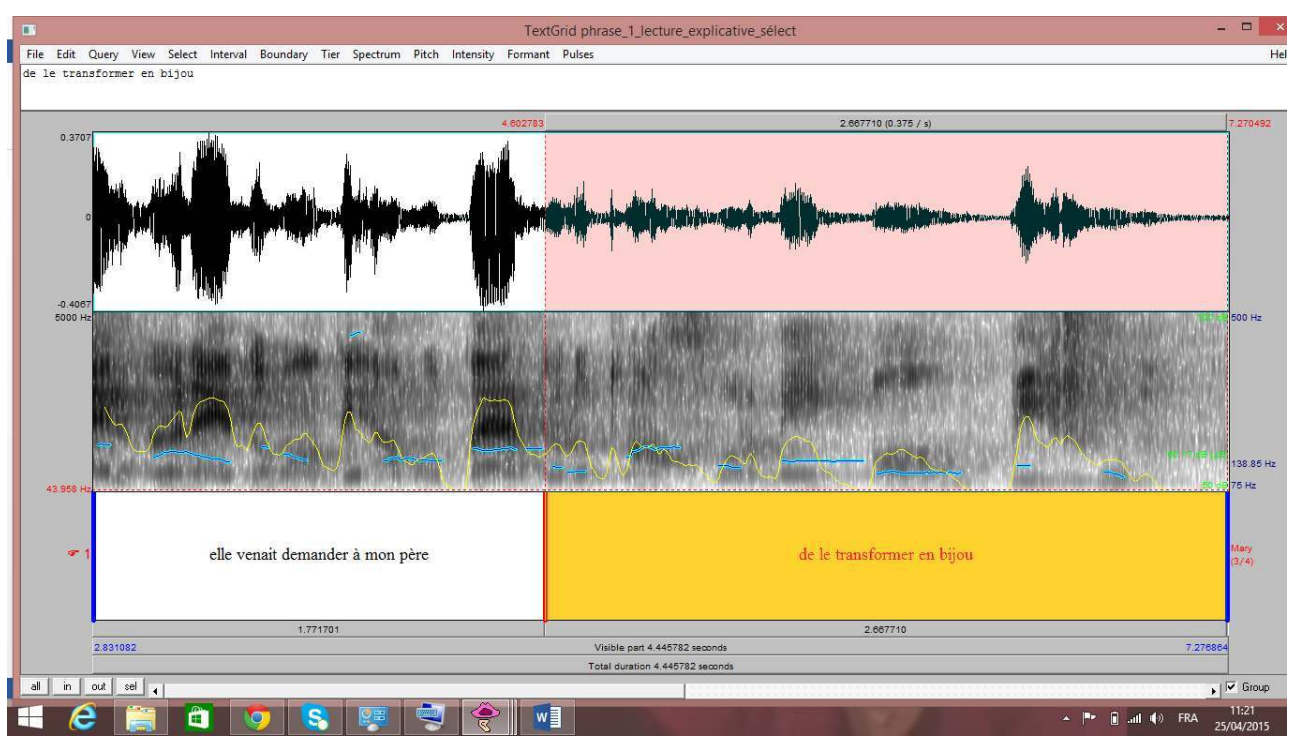

Fonagy (1991 : 109) estime que l'accent est un «élément indispensable à la communication d'idées conscientes, d'un outil verbal de haute valeur ». Lors de l'activité de la hiérarchisation des proéminences, un choix s'opère sur l'axe syntagmatique pour mettre en relief, au moyen de l'accent, l'unité la plus importante de l'énoncé, comme pour le mot «bijou » prononcé avec un accent de durée (Tableau 3). Faire prononcer la réponse correcte après une question sur ce qui a été lu est donc facilité par un usage vocal précis : la focalisation. Les outils prosodiques utilisés (Tableau 3) diffèrent d'un enseignant à un autre. 
Tableau 3 - Les caractéristiques prosodiques de la partie focalisée (transformer en bijou)

\begin{tabular}{|l|l|}
\hline $\begin{array}{l}\text { Partie focalisée } \\
\text { Lecture explicative }\end{array}$ & Outils prosodiques utilisés pour focaliser \\
\hline transformer en BI-JOU & $\begin{array}{l}\text { - ralentissement du débit et allongement des syllabes } \\
\text { - prononciation séparée des deux syllabes constituant le mot « bijou ». }\end{array}$ \\
\hline
\end{tabular}

La compatibilité entre la partie lue focalisée «le transformer en bijou » et la question posée «que vient faire la femme chez le père du narrateur?» a facilité la discrimination de la réponse par les apprenants (Tableau 4).Le degré de compatibilité entre la partie focalisée lors de la lecture explicative et la question posée nous renseignent sur le degré de conscience de l'enseignant de l'influence de son usage vocal (focalisation) sur l'orientation des apprenants vers la réponse souhaitée.

Tableau 4 - La compatibilité entre la partie focalisée, la question posée et la réponse saisie

\begin{tabular}{|l|l|l|l|}
\hline $\begin{array}{l}\text { La partie } \\
\text { focalisée }\end{array}$ & La question posée sur la phrase lue & $\begin{array}{l}\text { La réponse } \\
\text { directe } \\
\text { des apprenants }\end{array}$ & $\begin{array}{l}\text { Les indices de } \\
\text { référence pour la } \\
\text { réponse }\end{array}$ \\
\hline $\begin{array}{l}\text { transformer en } \\
\text { BI-JOU }\end{array}$ & $\begin{array}{l}\text { que vient faire la femme ? que venait } \\
\text { faire la femme } \uparrow+\text { chez } \uparrow+\text { le père } \\
\text { du narrateur +? }\end{array}$ & $\begin{array}{l}\text { Elle transformait } \\
\text { en bijou }\end{array}$ & $\begin{array}{l}\text { toute la partie } \\
\text { focalisée par la } \\
\text { prosodie r a }\end{array}$ \\
\hline
\end{tabular}

Les apprenants (de niveau moins avancé) ont repris la partie focalisée telle quelle est « transformer en bijou » sans réfléchir à la structure grammaticale où il faut au verbe " transformer» un complément d'objet direct: "l'or». C'est peut-être parce que le mot «or » ne figure pas dans la partie mise en relief par la prosodie de l'enseignant en lisant la phrase «elle venait demander à mon père de le transformer en bijou ». La saisie de la réponse dépend du degré de compatibilité entre la partie focalisée dans la phrase lue et la question posée tout de suite après mais aussi de la discrimination par les apprenants d'une focalisation ou non. L'exemple s'inscrit à la fois dans le domaine pragmatique et sémantique de la parole où l'enseignant tend à agir par sa voix sur la perception des apprenants. Ces derniers sont amenés à saisir une préférence implicite.

\section{L'effet « pervers » de la focalisation prosodique lors de la lecture explicative}

Que l'enseignant évite de dire lui-même la réponse fait partie d'une stratégie préétablie mais qui est en même temps contrainte par la présence d'un observateur et d'un enregistreur de sa parole. La réponse donnée verbalement par l'apprenant révèle la réussite de l'orientation prosodique vers la réponse souhaitée. Dans la mesure où les apprenants se basent dans leurs réponses sur les indices prosodiques dans le discours de 
leur enseignant (en lisant ou en posant la question), nous pouvons dire que la prosodie de l'enseignant a un effet perlocutoire car elle agit sur le décodage de réponses probables. En effet, c'est la nature de la langue communiquée (langue étrangère) et le niveau moins avancé des apprenants qui fait que la parole des apprenants soit plus suscitée et " provoquée » qu'une parole libre et instantanée. Le but cognitif de l'enseignant se traduit par sa volonté de faire parler les apprenants en français mais aussi de les faire parler correctement.

31 Selon Cicurel(2011 : 19) «la classe de langue est un lieu dans lequel l'apprentissage s'effectue en partie du moins - par l'interaction». Cependant, si la réponse juste verbalisée ou prononcée témoigne en apparence de leur compréhension, elle ne prouve pas nécessairement que l'enseignant a su faire apprendre une information aux apprenants, elle ne confirme pas si effectivement ils ont compris pourquoi ils ont choisi telle ou telle réponse, car ils ont été orientés par l'intonation de l'enseignant focalisant les unités dominantes la phrase lue. Ainsi, sur le plan cognitif, il n'est pas sûr qu'une réponse verbale juste veuille dire que l'apprenant ait compris ce que l'enseignant voulait qu'il connaisse. Dans ce sens, la focalisation prosodique lors de la lecture explicative aura un effet pervers sur les réponses des apprenants.

\section{L'interaction verbale enchaînée par des indices vocaux et gestuels : analyse du corpus (2)}

L'enchaînement de l'interaction verbale en classe de FLE ne semble pas aléatoire ou être le produit de la spontanéité des paroles. Il ne s'agit pas d'une conversation ordinaire mais d'un un enchaînement "provoqué », suscité et orienté en grande partie par l'usage vocal et gestuel de l'enseignant. Pour transmettre un message interactionnel quelconque, l'enseignant produit les ligateurs et les ponctuantssuivants: «ah, bon, hein, oui». Un même ligateur ou ponctuant peut être compris différemment par les apprenants selon l'intonation avec laquelle il est produit et peut signifier des intentions de communications différentes et constitue des indices pour parler, parler mieux, autre, encore ou de ne plus parler.

\section{L'exemple de « Pardon » produit de manières différentes}

Afin de montrer le rôle de l'usage gestuel et vocal dans l'enchaînement de l'interaction verbale, le contrôle et l'orientation des réponses des apprenants, nous avons choisi le mot «pardon» utilisé par l'enseignante (corpus 2) dans des buts verbaux différents : faire parler, parler encore, parler mieux, parler haut et parler autre, etc.

\section{« Pardon » pour désigner et faire parler un apprenant}

Désigner un apprenant pour qu'il prenne la parole se fait généralement de manière différente soit en prononçant le prénom de l'apprenant, soit par le regard seul, soit par le mot «oui» accompagné du regard, etc. La forme gestuelle et vocale dont fait usage l'enseignante consiste à produire le mot «pardon» en désignant par le doigt et le regard l'apprenant concerné (Image 1) : 
Figure 3 - Le geste accompagnant le mot « pardon » désignant un apprenant pour parler

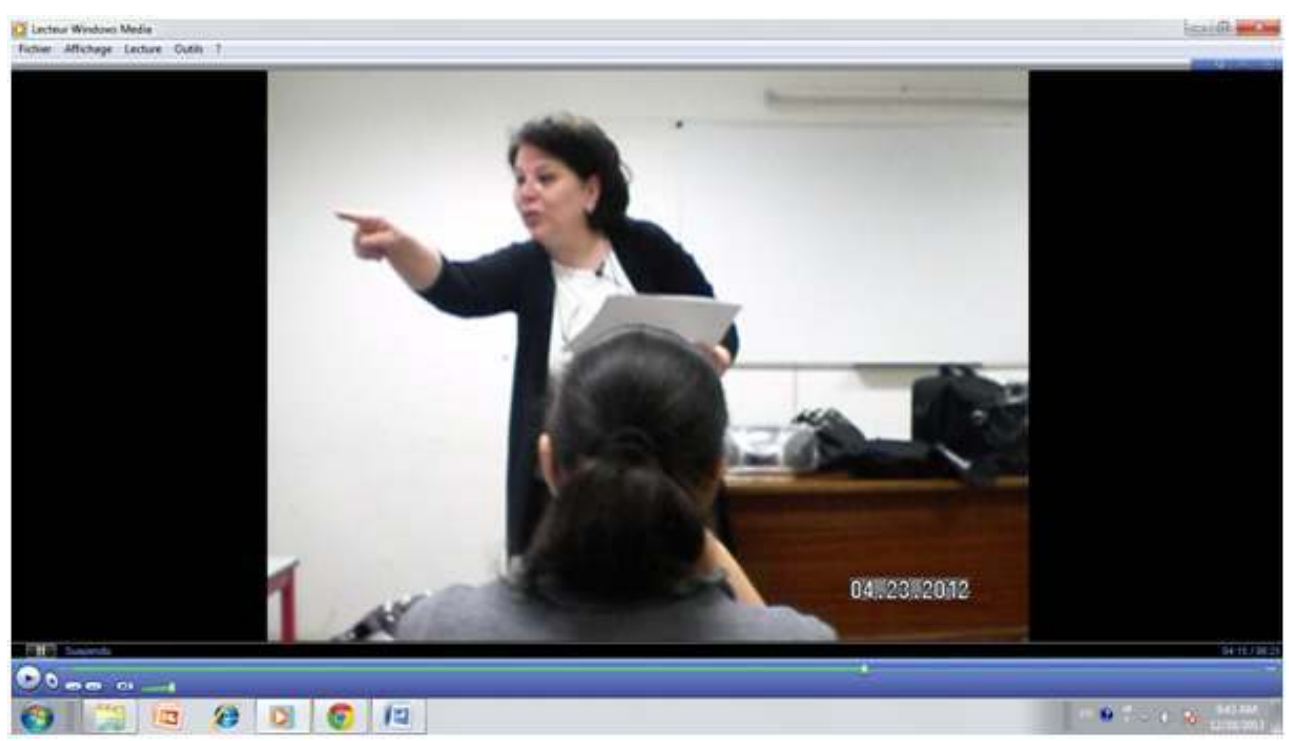

Plusieurs apprenants à la fois tentaient de donner des réponses et l'enseignante a choisi de donner la parole à un apprenant dont la réponse était juste.

L'intonation avec laquelle est prononcé le mot «pardon» (fréquence particulièrement haute de la deuxième syllabe «don» Tableau 8) révèle l'intention de l'enseignante que l'apprenant réitère sa réponse. Ce "pardon » n'a pas été commenté par l'enseignante, pour ce fait nous reprenons dans le Tableau 5 uniquement le contenu de l'interaction.

Tableau 5 - «Pardon » pour faire parler en reprenant sa réponse

\begin{tabular}{|l|}
\hline INTERACTION (Séquence 2) \\
\hline 07A populaire \\
08 P Pardon $\uparrow$ \\
09A Populaire
\end{tabular}

37 L'apprenant, comprenant l'intention de son enseignante et le message communiqué, reprend la même réponse qu'il a produite : « populaire ».

38 Il existe un lien entre le fait que les apprenants reprennent et confirment leur réponse et l'appréciation positive qu'ils ont saisie. Sur le plan de l'analyse la parole, Caelen Haumont précise que la fonction du « faire croire » prosodique indique :

comment l'énoncé produit par le locuteur est appréhendé par celui-ci et à la fois comment l'auditeur doit saisir le sens qui lui est donné, ce dernier restant libre, bien entendu, d'adhérer ou non à ce point de vue (Caelen Haumont, 2005 :193). Dans un contexte didactique, l'apprenant n'est pas libre d'accepter ou d'ignorer l'évaluation perçue dans l'intonation et le geste de l'enseignant, il est amené à modifier ou à confirmer sa réponse suite aux indices perçus d'évaluation positive ou négative.

40 Sur le plan de l'analyse du geste pédagogique (GP), Tellier $(2008 ; 2014)$ distingue les fonctions d'informer, d'animer et d'évaluer. Nous pensons que pour pouvoir enchaîner l'interaction verbale, l'enseignante, en désignant un apprenant parmi plusieurs 
apprenants qui ont répondu en même temps pour parler, effectue une évaluation positive implicite de la réponse prononcée préalablement par l'apprenant, c'est un indice l'informant que sa réponse est juste et qu'il doit la reprendre telle qu'elle.

\section{« Pardon » pour faire parler haut}

Nous avons remarqué que les apprenants reprennent et confirment leur réponse après une appréciation positive de l'enseignant qui les rassure que leur réponse est dans la bonne voie, et qui, par la suite, agit sur l'intensité de leur voix. Ils augmentent l'intensité de leur voix suite à une appréciation implicite ressentie et perçue dans la voix de leur enseignant. Selon Cicurel (2011: 117), «l'agir peut être connu à la fois par l'observation des interactions et par les dires des acteurs à propos de l'action». Le Tableau 6 montre à gauche le contenu de l'interaction et à droite celui de l'auto-commentaire de l'enseignante. Rappelons que cette dernière est amenée à s'auto-commenter tout en visionnant le film.

La « mise en mots » de l'action s'inscrit dans un agir dans la mesure où il y a intentionnalité et actions exécutées "subordonnées à un but global » (Cicurel, 2011: 119). Le mot " pardon» a été utilisé en toute connaissance de cause par l'enseignante dans le but d'agir sur la voix et le moral de l'apprenant (Tableau 6).

Tableau 6 - Autocommentaire où « pardon » signifie « parler haut »

\begin{tabular}{|l|l|}
\hline $\begin{array}{l}\text { INTERACTION } \\
\text { (Séquence 1) }\end{array}$ & ENTRETIEN D'AUTO-CONFRONTATION \\
\hline $\begin{array}{l}\text { A c'était un } \\
\text { voleur } \\
03 \text { PPardon } \uparrow \\
04 \text { A c'était un } \\
\text { voleur }\end{array}$ & $\begin{array}{l}\text { P j'ai fait répéter réellement parce que j'ai pas entendu mais j'ai aussi } \\
\text { lesoin de de lui donner un tout petit peu plus d'assurance pour qu'on } \\
\text { l'entende }\end{array}$ \\
\hline
\end{tabular}

L'outil gestuel utilisé par l'enseignante en prononçant le mot «pardon» consiste à toucher l'oreille avec la main droite et à regarder en direction de l'apprenant concerné (Image 2) :

Figure 4 - Le geste accompagnant le mot « pardon » pour amener à parler plus fort

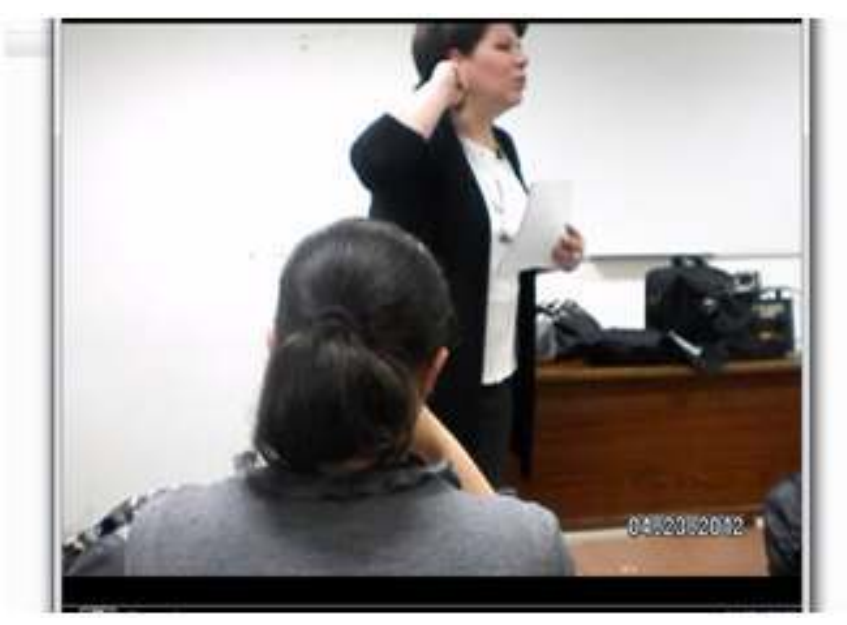



voleur " mais avec une intensité plus forte. La reprise par l'apprenant de sa réponse (Tableau 6/Interaction) est encouragée par le geste corporel et le comportement vocal de l'enseignante en produisant le mot "pardon", la deuxième syllabe étant fortement allongée (Tableau 8). L'intention verbalisée "j'ai aussi besoin de de lui donner [...] l'entende " (Tableau 6/ Entretien) a donc bien été saisie par l'apprenant grâce à deux indices simultanés : la voix et le geste. Sur le plan interactionnel, l'apprenant reprend la même réponse quand l'intonation est appréciative (évaluation positive).

\section{« Pardon » pour faire parler mieux}

Le même mot "pardon" est prononcé cette fois pour "faire parler mieux" pour que l'apprenant prenne conscience de son erreur » (Tableau 7/Entretien) :

Tableau 7 - Autocommentaire où « pardon » signifie « parler mieux »

\begin{tabular}{|l|l|}
\hline INTERACTION (séquence 2) & ENTRETIEN D'AUTO-CONFRONTATION \\
\hline $\begin{array}{l}\text { 11A familial } \\
\text { 12P pardon } \uparrow \\
\text { 13A familier }\end{array}$ & $\begin{array}{l}\text { P j'ai fait répéter pardon simplement pour qu'elle } \\
\text { prenne conscience de son erreur et pour qu'elle } \\
\text { s'auto-corrige je suis aussi dans l'auto-correction. }\end{array}$ \\
\hline
\end{tabular}

Être désigné par le doigt a permis à l'apprenant de prendre la parole, mais c'est la mimique du visage de l'enseignante (sourire avec les dents serrées / Figure 3) qui a fait comprendre à l'apprenant qu'elle n'était pas totalement satisfaite de la réponse.

\section{Figure 5 - Le geste accompagnant le mot « pardon » pour faire « parler mieux »}

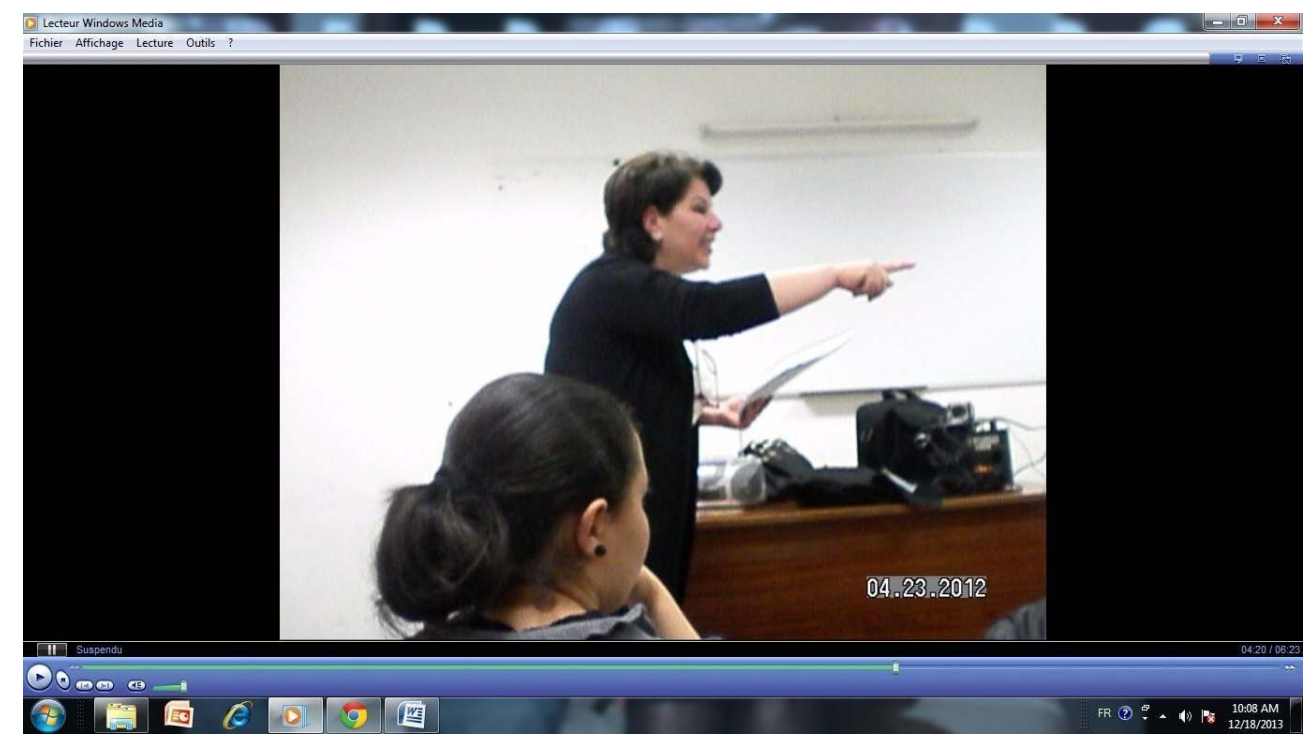

Selon Tellier (2008: 42), avoir recours au GP (geste pédagogique) sert plutôt à « indiquer un problème dans la réponse de l'élève » et "non à donner la bonne réponse ». L'apprenant reprend sa réponse en l'ajustant «familier» au lieu de «familial », car il a compris, à 
travers l'intonation de l'enseignante, mais aussi grâce à la mimique de son visage, une demande de « parler mieux».

\section{Comparaison des réalisations acoustiques et gestuelles de «pardon »}

En comparant les trois réalisations du mot «pardon » (Tableau 8), nous remarquons que les valeurs intonatives de la syllabe «don » ne sont pas les mêmes ainsi que les usages gestuels.

durée et la fréquence sont élevées quand la réponse est juste et les deux sont assez basses quand le cas contraire. Dans le premier cas, l'enseignante demande à l'apprenant de confirmer sa réponse, de parler plus fort, et dans le $2^{\text {ème }}$, elle lui demande de parler mieux.

Le mot "pardon" dans les trois reprises fonctionne comme les «ligateurs " et les « ponctuants» comme «bon, hein, ah, oui» (Morel \& Boileau, 1998) qui servent à communiquer l'intention du locuteur selon l'intonation adoptée. L'apprenant a repris et confirmé sa réponse "populaire » dans le premier cas, il a confirmé sa réponse "c'était un voleur» avec une intensité plus forte dans le deuxième, et il a modifié et corrigé sa réponse («familier » au lieu de « familial ») dans le troisième cas.

Tableau 8 - Les trois manières de produire le mot « pardon » et les indices saisis par les apprenants

\begin{tabular}{|c|c|c|c|c|}
\hline $\begin{array}{l}\text { Les trois } \\
\text { pardons }\end{array}$ & $\begin{array}{l}\text { Durée de l syllabe } \\
\text { «don» } \\
\text { millisecondes }\end{array}$ & $\begin{array}{l}\text { Fréquence de la } \\
\text { syllabe «don » } \\
\text { en } \mathrm{Hz}\end{array}$ & Geste associé & $\begin{array}{l}\text { Indice saisi par les } \\
\text { apprenants }\end{array}$ \\
\hline $\begin{array}{l}\text { Pardon } \\
\text { Pour faire } \\
\text { parler }\end{array}$ & 170 & 489 & Désigner par le doigt & $\begin{array}{l}\text { Reprendre la } \\
\text { même réponse } \\
\text { «populaire» }\end{array}$ \\
\hline $\begin{array}{l}\text { Pardon } \\
\text { Pour faire } \\
\text { parler fort }\end{array}$ & 510 & 375 & La main à l'oreille & $\begin{array}{l}\text { Parler plus fort } \\
\text { confirmer la } \\
\text { réponse } \\
\text { «c'était un voleur» }\end{array}$ \\
\hline $\begin{array}{l}\text { Pardon } \\
\text { Pour faire } \\
\text { parler } \\
\text { mieux }\end{array}$ & 26 & 360 & $\begin{array}{l}\text { Désigner par le doigt } \\
\text { avec un sourire et } \\
\text { des dents serrées }\end{array}$ & $\begin{array}{lr}\text { Parler } & \text { mieux, } \\
\text { corriger } & \text { la } \\
\text { réponse } & \\
\text { «familial familier » }\end{array}$ \\
\hline
\end{tabular}

51 Nous pouvons enfin imaginer que d'autres mots ont des fonctionnements pragmatiques différents et stabilisés, en fonction de leurs propriétés acoustiques et des gestes associés, comme « hein » par exemple qui a déjà été étudié par d'autres chercheurs comme Léglise et al. (2006).

52 Le comportement vocal et prosodique de l'enseignant de langue étrangère fait donc partie de son agir en donnant son cours de langue en classe. Il agit sur l'enchaînement de l'interaction verbale en quantité et en qualité en multipliant les productions verbales des 
apprenants et en les orientant vers la production verbale satisfaisante (la réponse souhaitée).

\section{L'indice d'insatisfaction pour faire parler mieux et autre}

En observant la manière dont l'enseignant reprend la réponse des apprenants avec un indice d'insatisfaction (2191), nous avons remarqué que ces derniers multiplient et modifient leurs réponses comme pour l'exemple du mot «ils» (Tableau 9) qui a été perçu par les apprenants sous forme d'une demande de « parler encore».

Tableau 9 - Multiplication des réponses des apprenants face à un indice d'insatisfaction

\begin{tabular}{l} 
INTERACTION (séquence 3 ) \\
\hline 03 P ils $\uparrow . .$. \\
04 A ils exploitent le problème \\
05 P i i ils ils $\uparrow . .$. \\
06 A ils dévoilent \\
07 P ils dévoilent ils $\uparrow . .$. \\
08 A xx \\
09 A ils militent
\end{tabular}

54 Par contre, la reprise par l'enseignant de la totalité de la réponse avec un indice de satisfaction $(\downarrow)$ permet aux apprenants de comprendre la demande de ne plus parler sur le sujet (Tableau 10).

Tableau 10 - Indice de satisfaction qui induit le silence chez les apprenants

\begin{tabular}{|l|l|l|}
\hline $\begin{array}{l}\text { INTERACTION (séquence } \\
2)\end{array}$ & Indice saisi & Réaction verbale de l'apprenant \\
Reprise de la réponse & & \\
\hline $\begin{array}{l}19 \text { A social } \\
20 \text { P social } \downarrow++\end{array}$ & $\begin{array}{l}\text { Satisfaction de la } \\
\text { réponse }\end{array}$ & $\begin{array}{l}\text { Ne proposent plus de réponses pendant la } \\
\text { pause }(++)\end{array}$ \\
\hline
\end{tabular}

Une certaine évaluation de la réponse est donc perçue grâce à l'intonation accompagnant la reprise de la réponse des apprenants: une continuation de l'intonation $(\uparrow)$ avec une demande de parler encore quand la réponse est insatisfaisante et une conclusion de l'intonation $(\downarrow)$ avec une demande de ne plus parler sur le sujet quand la réponse est satisfaisante. 


\section{Ritualiser ses usages vocaux et gestuels : vers un contrat interactionnel} explicative encourage les apprenants à prendre la parole, il a un effet pervers sur leurs réponses car il montre indirectement le contenu de la réponse souhaitée. Le second exemple étudié (les différents usages vocaux et gestuels d'un même terme "pardon ») a montré le rôle des indices intonatifs et gestuels dans l'enchaînement de l'interaction verbale. L'apprenant saisit des demandes différentes selon la manière dont l'enseignant produit et illustre par des gestes le mot "pardon» (parler, parler mieux, parler haut, parler encore, parler autre etc.). Suite aux demandes saisies, l'apprenant reprend sa réponse, la modifie ou l'enrichit, etc. Les outils prosodiques et gestuels utilisés en vue de réaliser les objectifs (faire parler et prononcer la bonne réponse) relèvent d'un profil interactionnel spécifique et dépend de la volonté de chaque enseignant d'agir par sa voix et par ses gestes.

$61 \mathrm{Si}$, d'un côté, l'apprenant est souvent « dans la position de celui qui « répond » à un participant qui «l'interroge » » (Cicurel, $2011:$ 30), les indices prosodiques font partie des «amorces $d u$ prof [qui] balisent "pré-façonnent" la parole de l'apprenant" (Cicurel, 1994: 112) et constituent un facteur déclencheur de paroles en incitant les apprenants à parler en langue étrangère. Mais si, d'un autre côté, l'apprenant compte entre autres sur la manière de parler et les gestes de son enseignant pour trouver la réponse correcte à une question 
de compréhension, des réflexions peuvent être lancées sur l'impact (positif ou négatif) des indices prosodiques et gestuels sur l'autonomie de l'apprenant.

\section{BIBLIOGRAPHIE}

Caelen Haumont, G. (2006). Prosodie et sens : une approche expérimentale. Marges Linguistiques.

M.L.M.S. Publisher. pp.135-272.

Cicurel, F. (1985). Paroles sur paroles ou le métalangage en classe de langue. Paris : CLE.

Cicurel, F. (1994). « Discours d'enseignement et discours médiatiques : pour une recherche de la didacticité ». Les Carnets du Cediscor, vol. 2. pp. 93-108.

Cicurel, F. (2002). « La classe de langue un lieu ordinaire, une interaction complexe ». AILE, $\mathrm{n}^{\circ} 16$. pp. 145-164.

Cicurel, F. (2011). Les interactions dans l'enseignement des langues, agir professoral et pratiques de clases. Paris : Didier.

Colletta, J.-M. (2005). « Communication non verbale et parole multimodale: quelles implications didactiques? ». Le Français dans le monde, Recherches et applications. pp.32-41. Consulté de http:// edutice.archives-ouvertes.fr/edutice-00120446.

Fonagy, I. (1991). La vive voix : essais de psycho-phonétique. Paris : Payot.

Guimbretière, E. (2000). Apprendre, enseigner, acquérir : la prosodie au cœur du débat. Rouen : Presses Universités de Rouen-Havre.

Gumperz, J. (1989). Engagez la conversation. Paris : Les Éditions de Minuit.

Morel, M.-A. \& Danon Boileau, L. (1998). Grammaire de l'intonation. Paris : Ophrys.

Morel, M.-A.(2001/2002). «Intonation et gestion du sens dans le dialogue oral en français ». Intonation et Genres. Le statut de l'écouteur. Paris : Centre de Polycopiés de l'Université Sorbonne Nouvelle - Paris 3. pp. 203-220.

Moustapha Sabeur, M. (2008). La voix de l'enseignant et la communication de la langue étrangère, étude des facteurs influents liés au passé et au contexte présent. Thèse de doctorat, mention très honorable à l'unanimité. Université Sorbonne Nouvelle - Paris 3.

Moustapha Sabeur, M. (2010). « Usage de la voix par un enseignant de français langue étrangère lors de la lecture explicative ». L'Information Grammaticale, n 127. pp.16-23.

Moustapha Sabeur M. \& Aguilar Rio J.(2014). « Faire corps avec sa voix : paroles d'enseignants ». In Tellier M. \& Cadet L. (dir.). Le corps et la voix de l'enseignant : une mise en contexte théorique et pratique. Paris : Maisons des Langues. pp.68-79.

Tellier, M. (2008). « Dire avec des gestes ». Le Français dans le monde, Recherches et applications, $\mathrm{n}^{\circ}$ 44. pp.40-50. 


\section{NOTES}

1. L'entretien d'auto-confrontation a été effectué selon la perspective introduite par le groupe de recherche IDAP (interactions didactiques et agir professoral) de l'université Sorbonne nouvelle Paris 3.

2. L'analyse a été effectuée avec Le logiciel Praat.

\section{RÉSUMÉS}

Dans une activité de compréhension écrite, la focalisation prosodique lors de la lecture d'un texte est un indice aidant les apprenants à détecter une mise en valeur implicite de la partie qui constitue l'objet d'un questionnement. L'indice prosodique, dans ce cas, s'avère être un facteur déclencheur de paroles mais qui a un effet pervers sur les réponses des apprenants. Cependant, l'indice gestuel et prosodique permet d'enchaîner l'interaction verbale en corrigeant l'orientation des réponses des apprenants. L'étude se base sur des théories en didactique, en analyse de la parole et en sociolinguistique interactionnelle. Des extraits de cours enregistrés et/ ou filmés sont analysés.

Prosodic focus while reading acts as an index for learners, whereby they are encouraged to speak and give out an answer, because they have detected implicitly the answer. The prosodic index in this case happens to be a trigger of words but has a perverse effect on learner responses. However, the gestural and prosodic index allows concatenating verbal interaction by correcting the orientation of learner responses. The study is based on theories of didactics, speech analysis and interactional sociolinguistics. Extracts from recorded and / or filmed classes are analyzed.

\section{INDEX}

Keywords : make speak, prosodic Index, gestural Index, interaction, prosodic focus, feedback Mots-clés : faire parler, indice prosodique, indice gestuel, interaction, focalisation prosodique, feedback

\section{AUTEUR}

\section{MALAK MOUSTAPHA SABEUR}

Université de Carthage, Tunisie ; EA2288 - DLTEC, IDAP, Université Sorbonne Nouvelle -Paris 3, France

Malak Moustapha Sabeur est maître assistante dans le département de Français à l'Université de Carthage - Institut Supérieur des Langues de Tunis. Elle est membre du laboratoire de recherche DILTEC, groupe de recherche IDAP (Interactions didactiques et pratiques de transmission) à l'Université Sorbonne Nouvelle - Paris 3. Ses principaux domaines de recherches sont : les 
interactions didactiques, la voix et la parole professorale et le rôle du non verbal en classe de français langue étrangère.

Courriel : malak.sabeur[at]yahoo.fr 